\title{
Innovative Approaches in Quality Management in Clinical Laboratories
}

\author{
Lina Souan, Maher A. Sughayer \\ King Hussein Cancer Center \\ Jordan
}

\section{Introduction}

Clinical laboratories must adhere to Good Laboratory Practices (GLP) to ensure the quality, integrity, and reliability of patient results. GLP principles include organization and personnel, a quality assurance program, facilities, equipments, reagents and materials, test systems, standard operating procedures, result reporting, and archiving of records and reports (Wikipedia,2010).

This chapter presents a review of the latest requirements in quality control and quality management in certified clinical laboratories. We will describe different stages in clinical testing procedures and highlight the critical steps that need to be monitored using quality assurance indicator tools. In addition, we will present our latest contribution to the field of quality control computer software which encompasses an innovative approach in monitoring quality control and quality assurance in laboratories.

Accumulating and analyzing quality control data as well as quality indicators help in developing the performance improvement plan in the laboratory. Performance improvement is a process which uses information from multiple sources to increase the probability of accomplishing desired outcomes to better serve the needs and expectations of patients. The most frequently used performance improvement tools are: 1. FOCUS-PDCA framework for improvement, 2. Failure Modes and Effects Analysis (FMEA), 3. Root Cause Analysis (RCA) and 4. Control Charts. However, in this chapter we will focus on one model of quality improvement approaches which is FOCUS-PDCA and control charts and give examples on its implementation in a clinical laboratory setting.

\section{Imperative}

At the end of this chapter, you will be able to understand different stages of analytical testing; differentiate between internal and external controls, analyze common problems associated with invalid test results, have an overview of different QC software and our contribution to advance these programs.

Traditionally, medical laboratories used conventional paper-based systems for assessing quality of medical tests performed in the laboratory. The main drawbacks for these traditional quality control monitoring systems can be summarized in the following points.

Manual paper-based quality monitoring systems have a long lag time between tests and application of quality control procedures. The absence of a reporting system of the different 
system variables hinders the long-term planning and expansion of the system. Clinical laboratories that are still using these traditional ways of monitoring the quality control results have reduced reliability and productivity as there have been manually applying and assessing quality control rules such as Westgard rules (Al-Taee,2009).

Because of these problems and the desire to deliver high quality services, most of modern medical laboratories are planning to gradually transform all administrative and communicative work to become electronic. Hence, ample efforts are put in automating the quality control process in clinical laboratories in order to increase quality of the results. Several quality control programs are commercially available; however, we created a new program that captures the results entered by each medical technologist. The program analyzes the validity of each test, suggests corrective action(s) for failed quality control data as well as reports the number of failures in performing the daily quality control per each technologist. This not only confirms validity of the implemented quality control process, but delivers higher confidence in lab testing procedures, and allows for more reliable test results. Moreover, analyzing failure reports generated by the program which are related to poor performance enables the medical director in assessing the medical technologists' competency in addition to evaluating the performance of the instrument/ clinical analyzers. Furthermore, this data can be used in setting Quality Improvement plans in the laboratory (Al-Taee,2009).

\section{Quality management in clinical laboratories}

\subsection{Stages in performing analytical tests}

All analytical tests are divided into three stages: Pre-analytical stage, analytical stage and a post analytical stage.

\subsubsection{Pre-analytical stage}

The pre-analytical stage is the stage that precedes the tests analysis. This stage consists of several factors such as: Written policies and procedures, requisition for analysis, preparation of patient, patient identification, effective blood collection, mixing with anticoagulants, storage until transportation, transportation collection or courier service (Sharma,2009), and keeping accurate laboratory logs to ensure complete records of specimen arrival. These steps are performed by physicians, nursing staff, doctor's assistant and laboratory staff. Recent statistics have shown that $46-68.2 \%$ of total laboratory result errors are due to pre-analytical factors (Plebani,2006). Hence, controlling these steps by monitoring them and analyzing the data reduces the infidelity in lab test results.

\subsubsection{Analytical stage}

Analytical factors that influence the quality of laboratory results may include factors such as: Method of analysis, reagents used for the test, equipment, calibration, controls used in the test run, analysis of the control results using statistical programs, training and education of the laboratory technologist/technician. One of the important steps in the analytical stage is running quality control material.

Quality control (QC) refers to the routine technical activities that must be included during each assay run to verify that the test is working properly (Westgard, et al.,2006). The main purpose of running QC material is to ensure the validity of test method and test result, to 
prevent occurrence of errors by getting erroneous patient results, to identify the cause of errors and to correct errors if and when they occur.

The following steps should be followed in order to achieve the aforementioned goals of running QC material.

- Select the proper method for the analysis.

- $\quad$ Adopt the proper QC system

- Train the laboratory technologists on GLP practices and request that they get certification of training on the tests they perform.

- Keep all instruments calibrated and well maintained by performing the daily, weekly and monthly maintenance indicated by the manufacturer.

- Make sure that all reagents are handled and stored properly by closely monitoring the temperature in the refrigerators/ freezers and equipment during test analysis.

- Make sure that the technicians are following the written policies on properly handling and processing patient samples.

\subsubsection{Types of Quality Control}

Quality Control is divided into two categories: Internal and External Quality Control.

Internal Quality Control to the test kit is defined as control samples provided with the test kit with known reactivity.

External Quality Control to the test kit is defined as control samples not included with the test kit but provided from an external source that has been validated for use with a specific test kit.

\section{Invalid Quality Control:}

In an event that the Quality Control results were invalid, then follow one or more of the following corrective actions:

- Repeat test

- If repeatedly invalid:

- $\quad$ assume a problem with test product or procedure

- continue with alternative testing algorithm

- Identify cause of problem

- Inform supervisor

- Take corrective actions

Table 1- presents examples of some of the problems encountered while performing controls of serological tests and the proposed actions that should be taken to correct theses problems. Records of internal control results must be periodically reviewed. These records should be reviewed before accepting test results. As for the external control results, they should be reviewed by test performer as well as reviewed weekly or monthly by testing site supervisor.

\section{External Quality Control:}

External Quality Control samples can be either prepared by a "Reference Laboratory" or commercially prepared.

The frequency of using test External Control samples could be as follows:

- $\quad$ Minimum once a week and preferably at the beginning of the week

- New shipment of test kits

- Starting a new lot number

- If environmental conditions exceeded range needed for stability of kits. 


\begin{tabular}{|l|l|l|}
\hline Problem & Potential Cause & Action \\
\hline $\begin{array}{l}\text { No control line or band } \\
\text { present }\end{array}$ & $\begin{array}{l}\text { Damaged test device or } \\
\text { controls }\end{array}$ & $\begin{array}{l}\text { Repeat the test using new } \\
\text { device and blood sample. }\end{array}$ \\
\hline followed & $\begin{array}{l}\text { Follow each step of testing } \\
\text { according to the written } \\
\text { standard operating procedure. } \\
\text { Re-check buffer and/or } \\
\text { specimen volumes. } \\
\text { Wait for the specified time } \\
\text { before reading the test. }\end{array}$ \\
\hline $\begin{array}{l}\text { Positive reaction with } \\
\text { negative external control, } \\
\text { i.e. false positive }\end{array}$ & Expired or improperly \\
\hline $\begin{array}{l}\text { Extremely faint control } \\
\text { line }\end{array}$ & controls & $\begin{array}{l}\text { Check expiration date of kits or } \\
\text { controls. Do not use beyond } \\
\text { stated expiration date } \\
\text { Check temperature records for } \\
\text { storage and testing area }\end{array}$ \\
\hline
\end{tabular}

Table 1. Potential causes of problems encountered while running QC \& corresponding corrective action(s).

In summary, using internal and external quality control insures that the reagent material and solutions involved in the assay procedure did not deteriorate after validation, and they can be incorporated in the run one or more times every run of analysis. Data from running control material is plotted on control charts and interpreted following what is called Westgard Statistical rules (Thompson,2010).

\subsubsection{Statistical QC Rules}

\section{a. Arithmetic Mean or average}

The arithmetic mean of a group of numbers is found by dividing their sum by the number of members in the group (BioRad,1995). For example, the sum of the four numbers 3, 4, 5 and 20 is 32; hence, their mean is 32 divided by 4 , which comes up to 8 . In clinical laboratories, the arithmetic mean is often called the target value.

Note that when control material is assayed the values of the controls rarely fall on the target mean. The values rather show distribution around the mean.

\section{b. Standard Deviation}

Standard Deviation is a measure of the dispersion of a set of data from its mean. The more spread apart the data, the higher the deviation (BioRad,1995). The sample standard deviation is denoted by $s$ and defined as follows:

$$
s=\sqrt{\frac{1}{N-1} \sum_{i=1}^{N}\left(x_{i}-\bar{x}\right)^{2}},
$$




\begin{tabular}{|c|c|c|}
\hline Column A & Column B & Column C \\
\hline $\mathrm{x}$ values & $\mathrm{x}-\overline{\boldsymbol{x}}$ & $(\mathrm{x}-\overline{\boldsymbol{x}})^{2}$ \\
\hline 30 & $30-27.7=2.3$ & 5.29 \\
\hline 31 & $31-27.7=3.3$ & 10.89 \\
\hline 29 & $29-27.7=1.3$ & 1.69 \\
\hline 24 & $24-27.7=-3.7$ & 13.69 \\
\hline 28 & $28-27.7=0.3$ & 0.09 \\
\hline 33 & $33-27.7=5.3$ & 28.09 \\
\hline 20 & $20-27.7=-7.7$ & 59.29 \\
\hline 30 & $30-27.7=2.3$ & 5.29 \\
\hline 25 & $25-27.7=-2.7$ & 7.29 \\
\hline 27 & $27-27.7=-0.7$ & 0.49 \\
\hline$\sum \mathrm{x}=277$ & $\sum(\mathrm{x}-\overline{\boldsymbol{x}})=0$ & $\sum\left((\mathrm{x}-\overline{\boldsymbol{x}})^{2}\right)=132.1$ \\
\hline$\overline{\boldsymbol{x}}=27.7$ & & \\
\hline
\end{tabular}

Table 2. Example illustrating the calculation of the mean and standard deviation of a set of control data points.

Where $\mathrm{N}=$ group size, $\mathrm{x}=$ variable and $\bar{x}$ is the mean. Example:

- First we calculated the arithmetic mean of the group which is $\left(\sum\right)$ the sum of the individual points 277 then divided by 10 to be equal to 27.7 .

- Second, we subtracted the mean value from each control value (column B) and applied the following equation $x-\bar{x}$. As it is evident from table 1 that the difference can be a positive value or a negative value, however, the sum of the difference is always zero.

- $\quad$ Third, we squared the difference score to make all terms positive as seen in column C.

- Fourth, the squared difference was summed and used in the equation below to yield the predictable dispersion or standard deviation (SD or s).

$$
s=\sqrt{\frac{1}{N-1} \sum_{i=1}^{N}\left(x_{i}-\bar{x}\right)^{2}},
$$

$S=\left((132.1 /(10-1))^{1 / 2}=3.83\right.$

The value of the standard deviation allows us to predict the range of control values that should be observed if the method remains stable. From the example above, we can conclude the following:

- $\quad$ The range of the data is from 20-33

- $\quad$ The mean equals to 27.7

- $\quad \mathrm{SD}=3.83$ therefore one $\mathrm{SD}(1 \mathrm{SD})=3.83,2 \mathrm{SD}=7.66,3 \mathrm{SD}=11.49$

Note that all values of data in the example above are approximately within the range of the mean $\pm 2 \mathrm{SD}$. It is important to remember that the smaller the value of $\mathrm{SD}$ the better the result precision or reproducibility of the test.

\section{c. Coefficient of variation}

Coefficient of Variation (CV) is a measure of variability in a test or control level. It is expressed as a percent (BioRad,1995), and is calculated as:

$$
\mathrm{CV}(\%)=(\text { Standard deviation }(\mathrm{s}) \div \text { Mean })(100)
$$


Example: If the mean value for a control group was $117.4 \mathrm{IU} / \mathrm{L}$ and the SD was $5.03 \mathrm{IU} / \mathrm{L}$, then $\mathrm{CV}=(5.03 \mathrm{IU} / \mathrm{L} / 117.4 \mathrm{IU} / \mathrm{L})(100)=4.3 \%$

Calculating the $\mathrm{CV}$ for a test is useful in comparing manufacturer claims, College of American Pathologists (CAP) survey results, and peer group QC reports. It can also be used as a part of the internal quality control system when performing patient precision testing if the test materials used are similar and CVs are determined under similar conditions.

\section{d. Standard Deviation Index (SDI)}

It is important to participate in external proficiency testing program. These programs calculate the standard deviation index (SDI) and use it to compare a laboratory's results to its peer group. Furthermore, it allows the lab to gauge its performance when compared to the peer group. The SDI for the mean is calculated as follows:

$\mathrm{SDI}=($ mean of suspect data points - lab cumulative mean $) \div$ lab cumulative standard deviation.

The target SDI is zero. This would indicate that the lab's performance is identical to the peer group average. Acceptable SDI values are between $+/-1.0$. Any test/method/instrument which has a SDI between +/-1.0 and 1.5 may have a problem and the lab should investigate and troubleshoot and correct any test/ method/ instrument which has an SDI of $+/-2.0$ or greater. The lab can use the SDI statistic to interpret proficiency testing results (BioRad,1995).

\section{e. Median, Mode, and range}

- Median: The median is the middle number of a set of values arranged in an increasing order (Reynolds,2003). For example for the variables 4, 7, 3, 6, 2, 9, 11, the median number is $6(2,3,4,6,7,9,11)$. If you have an odd number of variables, the median is the middle number. But if you have an even number of variables, the median is the sum of the two middle values divided by 2 .

- Mode: The mode is the value which occurs most frequently (Reynolds,2003). For example the mode for the variables $3,5,5,7,7,7,9,10,10$, is 7 .

- Range: The range is the difference between the largest and the smallest variable in a distribution. It gives an indication of the dispersion of the data points, but it does not indicate how the data within the range vary about the mean. For example, the range for the variables $6,8,7,3,10,12,6,8,7,9,4$, is 3-12.

\section{f. Normal or Gaussian distribution}

Many of the control data accumulated will follow a certain distribution, with a peak around a certain value, and a few data points that lie outside the central cluster. This curve is called the normal distribution, the bell curve or the Gaussian distribution (Schieltz,2010). A frequency distribution curve is said to be normal when the curve of histogram is symmetrical bell shape. In this case, the mean, median and mode are identical. The figure below represents the frequency distribution of a large set of laboratory values obtained by measuring a single control material. This distribution shows the shape of a normal curve. In this figure we can notice the following points.

- $\quad$ Mean \pm 1 SD represents $68 \%$ of all values

- $\quad$ Mean \pm 2 SD represents $95 \%$ of all values (95\% confidence limit)

- Mean \pm 3SD represents $99.7 \%$ of all values.

At $95 \%$ confidence limit, it is obvious that $2.5 \%$ of the distribution is in the lower or left tail, and the same amount $(2.5 \%)$ is present in the upper tail. 


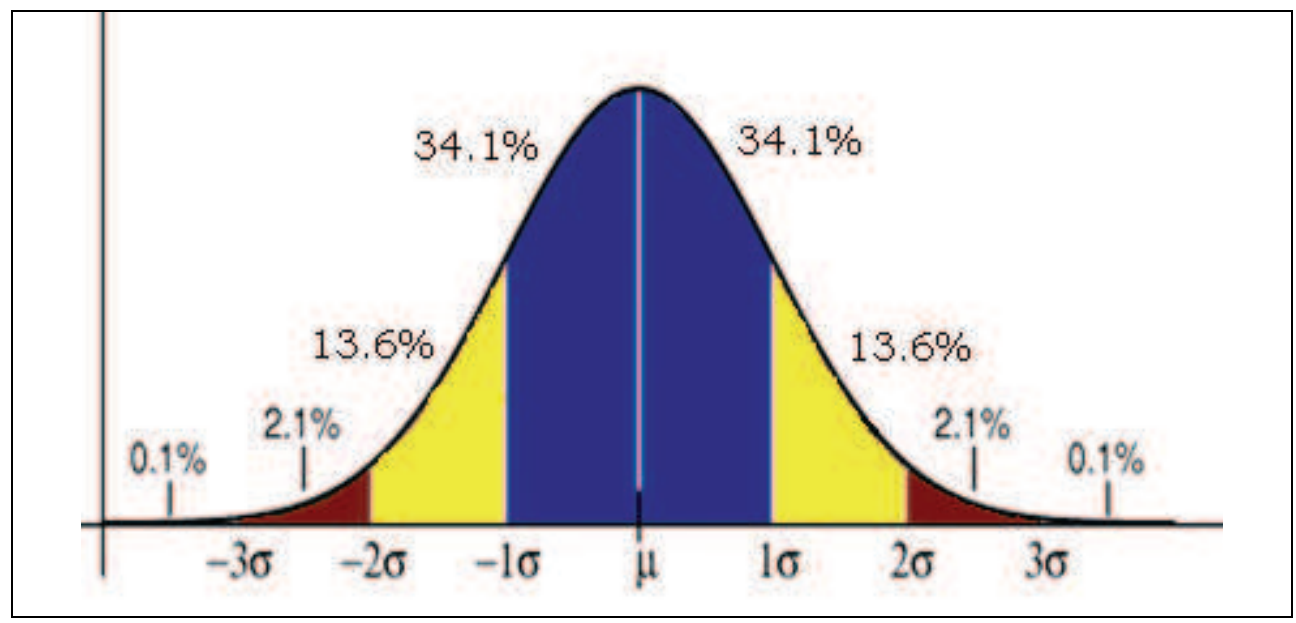

Fig. 1. Gaussian distribution illustrating, $68 \%$ of the values lie within 1 standard deviation of the mean; $95 \%$ lie within 2 standard deviations; and 99.7\% lie within 3 standard deviations.

\subsubsection{Statistical QC charts}

QC data should be evaluated for its accuracy and precision and a decision has to be made either to accept or reject the results before reporting patients' results. Therefore, several methods and actions must be taken in order to reach to the correct decision such as method validation (Lumsden,2000) and participating in an External Quality Assurance program. Other measures have to be taken in the laboratory in order to assure quality of work and results such as personnel training and education, instrument calibration and maintenance, good record keeping and finally plotting and reviewing of Quality Control Charts.

Quality Control Charts are a graphical representation of QC raw data used to evaluate whether a measurement procedure is in-control or out-of-control. Control results are plotted versus time or sequential run number; lines are generally drawn from point to point to highlight any trends, systematic shifts, and random errors (Westgard,2008).

A control chart must include control limits which are lines drawn on the chart to provide graphical criteria for assessing whether a measurement procedure is in-control or out-ofcontrol. These control limits are usually calculated from the mean and standard deviation (SD, or s) determined for a given control material. In general, interpreting the results is usually based on a specified number of results or points; when these data points are incontrol; patient test-results are reported thereafter. When the control data are out-of-control, the run must be rejected and no test results can be reported before the QC is repeated and is acceptable.

The most commonly used QC chart is the Levey-Jennings chart. The Levey-Jennings chart usually has the days of the month plotted on the X-axis and the control observations plotted on the Y-axis. By observing the data plotted in the Levey-Jennings chart, we can determine if test results are in control and accurate, or if test results are not in control and consequently unacceptable. The use of the Westgard, and Cumulative Summation Rules aids in establishing an effective error-detecting plan. The Levey-Jennings chart has horizontal lines at the mean value and at the control limits, usually 2 or 3 standard deviation. 
Manually constructed Levey-Jennings control charts used to be drawn by using graph paper having 10X10 or 20x20 lines per inch. One graph paper sheet is used for each level of the control. Two separate sheets must be used for a two control level because drawing both charts on a single sheet may reduce the readability of the control charts. The following steps are then are followed (Westgard,2002):

1. Label charts. Include the name of the test and the name of the control material in a top place so that this information is quickly and easily discerned when viewing the chart. The measurement unit in the following example is $\mathrm{pg} / \mathrm{mL}$, can be included in the label or included in the label for the y-axis. Other information typically included on the chart are the name of the analytical system, the lot number of the control material, the current mean and standard deviation, and the time period covered by the chart.

2. Scale and label $\mathrm{x}$-axis. The horizontal or $\mathrm{x}$-axis represents time and you will typically set the scale to accommodate 30 days per month or 30 runs per month. For this example, divide the $x$-axis into evenly sized increments and number them sequentially from 1 to 30. Label the x-axis "Day."

3. Scale and label y-axis. The y-axis represents the observed control value and you need to set the scale to cover the lowest and highest results expected. Generally the scale should cover a value as low as the mean - 4 standard deviations and a value as high as the mean +4 standard deviations (Westgard,2002). In this example, the chart for Control 1 should be scaled to accommodate a range from 200 to 64 , and 200 to 336.

4. Draw lines for mean and control limits. Mark the values that correspond to the mean and draw a black horizontal line (at $200 \mathrm{mg} / \mathrm{dL}$ for Control 1). Locate the values that correspond to the mean $+1 \mathrm{~s}$ and the mean $-1 \mathrm{~s}$ and draw blue horizontal lines (at 234 and 166 for Control 1). Locate the values that correspond to the mean $+2 s$ and the mean -2s and draw green horizontal lines (at 268 and 132 for Control 1). Locate the values that correspond to the mean $+3 \mathrm{~s}$ and the mean $-3 \mathrm{~s}$ and draw red horizontal lines (at 302 and 98 for control 1). Do the same procedure for Control 2 using the data of the mean and calculate SD as previously described and plot the data as shown in Figure 2.

\section{Quality Control Rules}

Control rule means a decision criterion for judging whether an analytical run is in-control or out-of-control. The rules for accepting or rejecting a run should be clearly stated in the quality control policy. For qualitative tests the QC rules are simple because the positive control must be positive and the negative control must be negative for the patient's results to be valid.

However, the quality control rules for the quantitative tests are set by the laboratory director. Some of the key points that the laboratory director should contemplate while setting the lab QC rules are: the medical necessity and precision of the test or assay used while setting the quality control limits, and refrain from choosing a single limit for all assays. Furthermore, the clinical significance of each test must be accounted for when evaluating QC limits and rules and the QC limits should be clearly stated in the QC policy manual and in the procedure manual. The most commonly used rule is the Levey-Jennings approach in which many laboratories choose 2 standard deviations on either side of the mean for their QC limits or rule. Since $95 \%$ of the values are covered by the 2 SD on either side of the mean in a normal distribution curve, the control will be out of limits in 1 out of 20 determinations by chance and any result exceeding 2SD values on either side of the mean is considered out of control (Westgard,2002). 

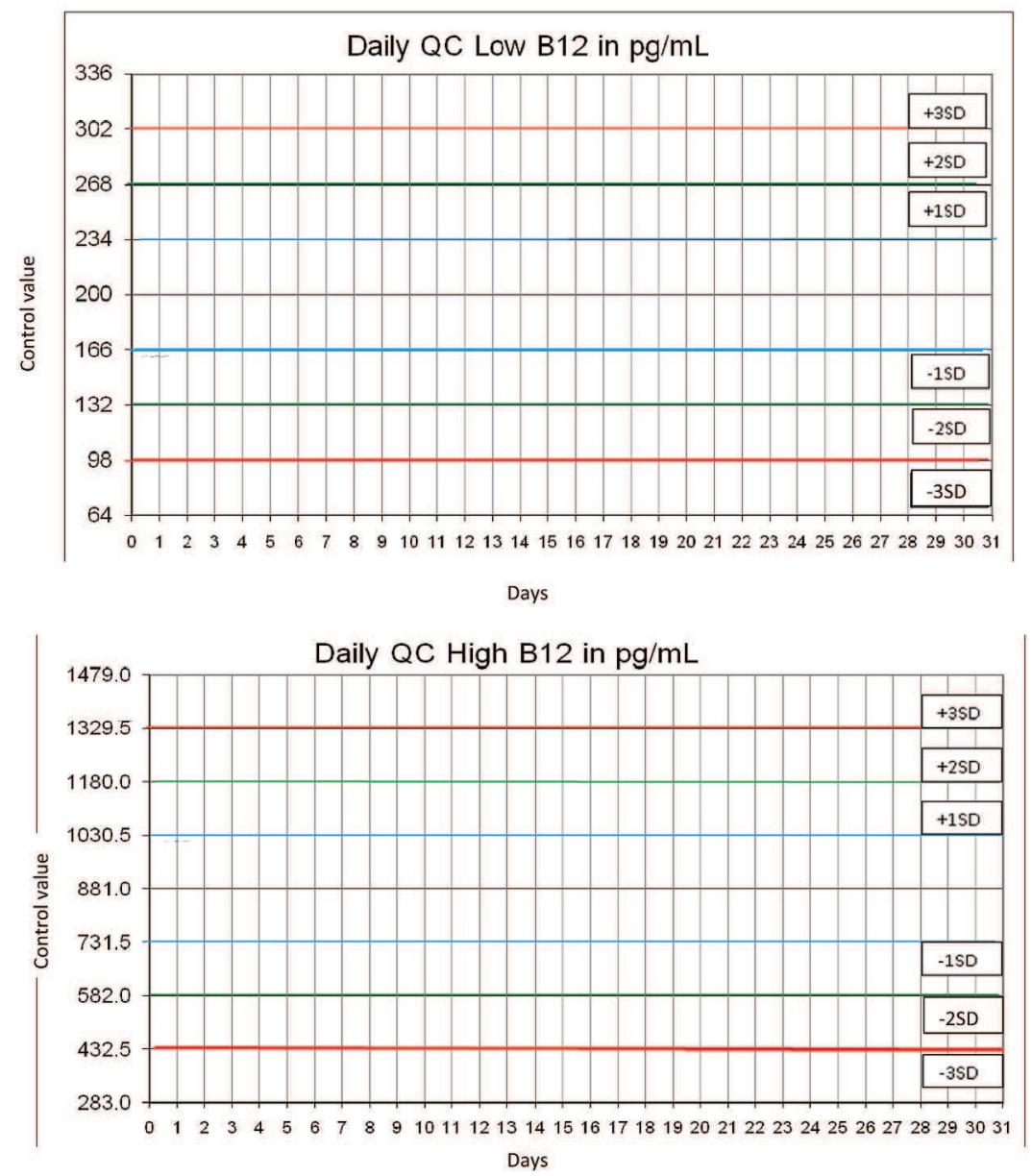

Fig. 2. A representation of Levey-Jennings chart with horizontal lines at the mean value and at the control limits, 1, 2 and 3 standard deviation.

\section{Westgard Rules}

It has been found that the false rejection rate is $5 \%$ when using one control level, and $9 \%$ when using two control levels while it goes up to $14 \%$ when using three control levels (Westgard,2002). In order to reduce the false rejection rate without compromising quality, Dr. James Westgard put some rules to analyze daily QC data.

Westgard rules are commonly used to analyse data in Levey-Jennings charts and to define specific performance limits for a particular assay. These rules can also be used to detect both random and systematic errors which will be explained later in this chapter. There are six commonly used Westgard rules of which three are warning rules and the other three are mandatory rules when two control levels are used.

The violation of warning rules should start a review of test procedures, reagent performance and equipment calibration. The violation of mandatory rules should result in the rejection of the results obtained with patients' samples in that assay (BioRad,1995). 
Dr. Westgard adopted a short hand notation to abbreviate different decision criteria or control rules, e.g., $1_{2 s}$ to indicate 1 control measurement exceeding 2 s control limits. Combinations of rules are generally indicated by using a "slash" mark (/) between control rules, e.g. $1_{3 s} / 2_{2 s}$.(Westgard,2009)

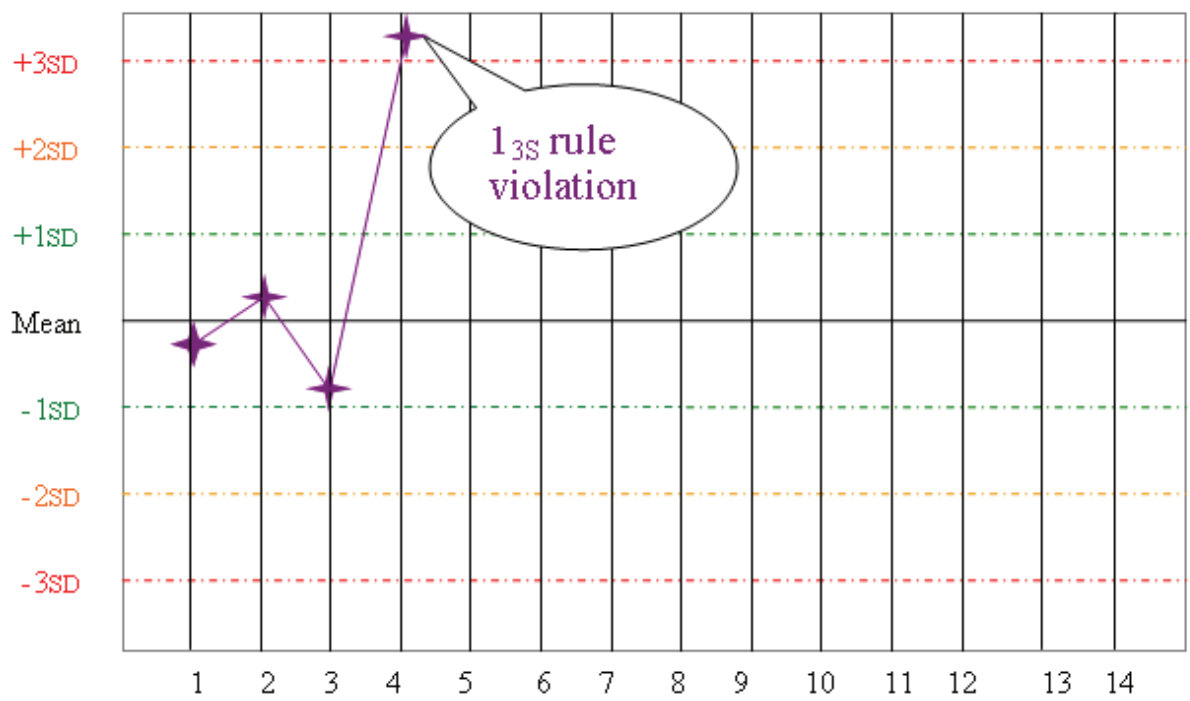

Fig. 3. Rule $1_{3 s}$ - refers to plus/minus $3 \mathrm{~s}$. A run is rejected when a single control exceeds the mean \pm 3 s.

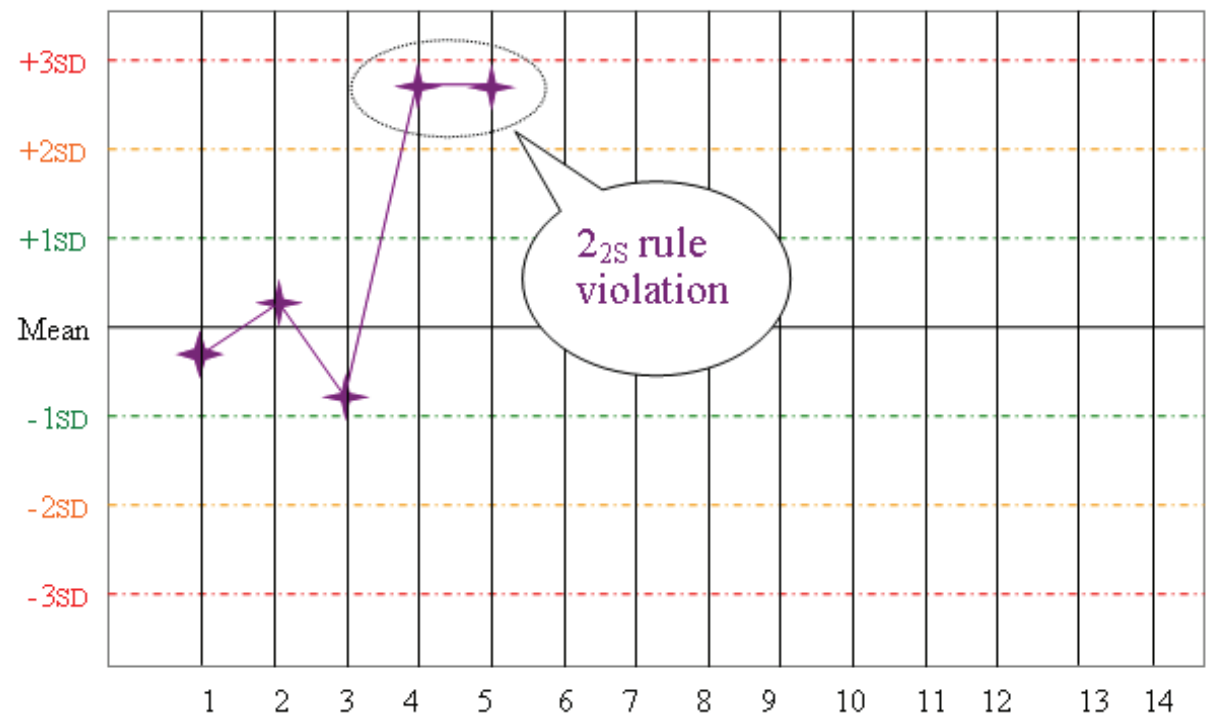

Fig. 4 . Rule $22_{2}$ - reject the run when 2 consecutive controls exceed the mean $\pm 2 \mathrm{~s}$. 


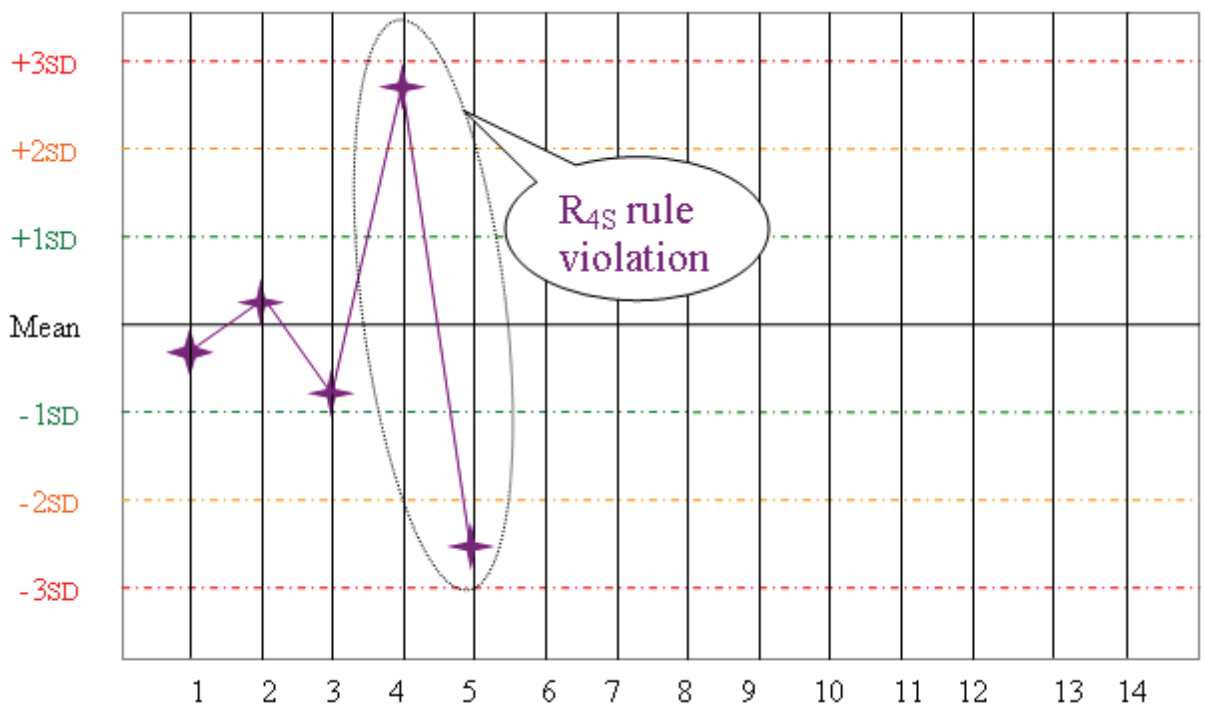

Fig. 5. Rule $\mathrm{R}_{4 \mathrm{~s}}$ - when 1 control in a group exceeds the mean $\pm 2 \mathrm{~s}$ and another control exceeds the mean in the other direction by 2 s, reject the run.

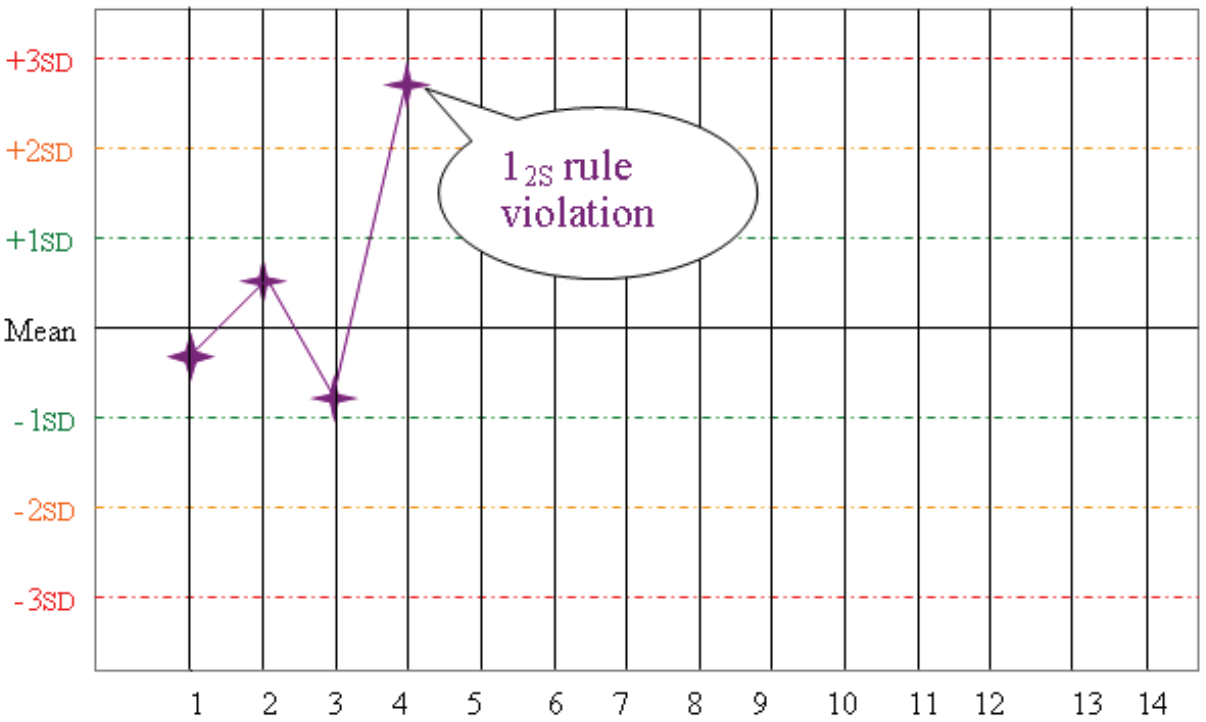

Fig. 6. Rule $1_{2 s}$ - refers to the historical rule of plus/minus 2 s from the mean, with multirules: a warning rule to trigger careful inspection of control data. 


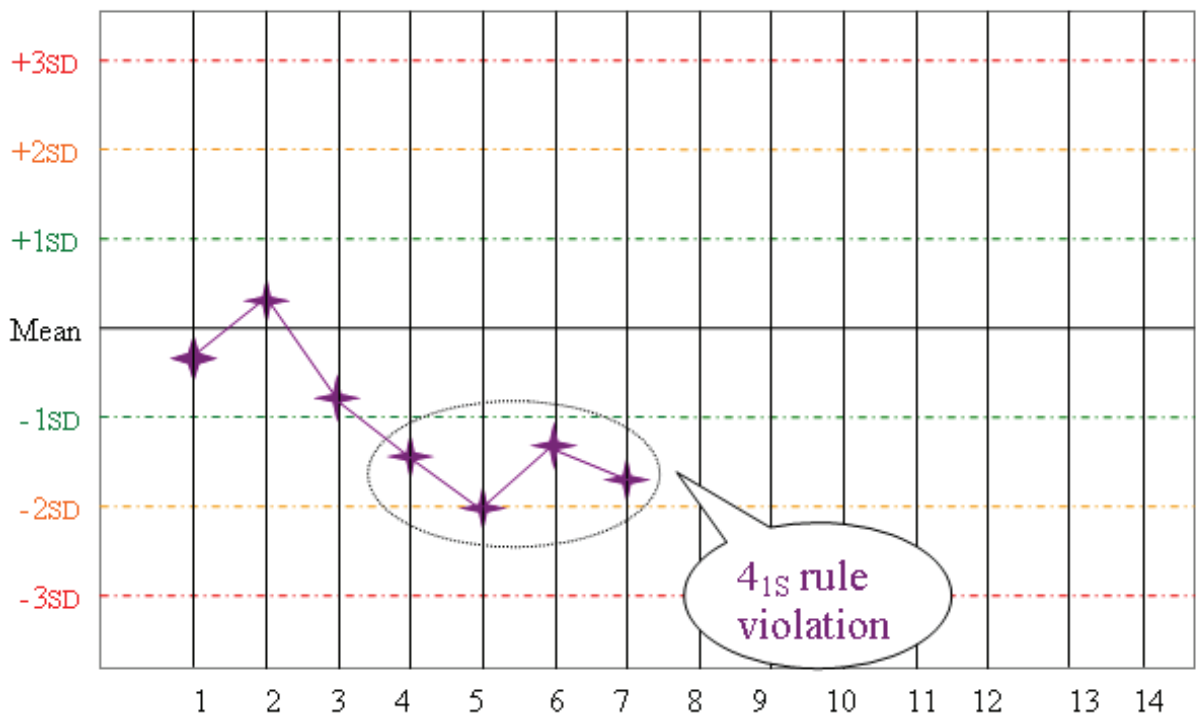

Fig. 7. Rule $41_{1 s}-$ when 4 consecutive control measurements are on one side of the mean either \pm 1 s. Warning rule or a rejection rule depending on the accuracy of your instrument.

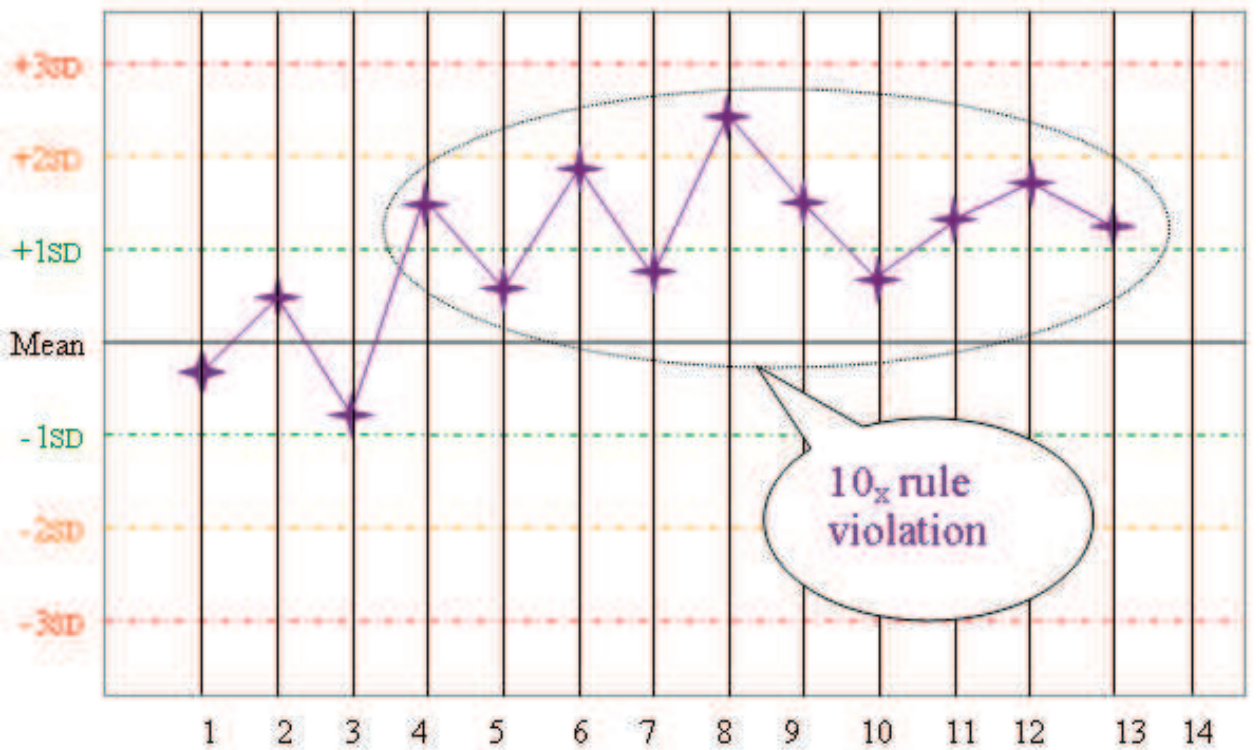

Fig. 8. Rule 10x - 10 consecutive control measurements fall on one side of the mean. If within $1 \mathrm{~s}$, warning. If between 1 and $2 \mathrm{~s}$, reject. 
When three different control level materials are being analyzed, some other control rules fit better and are easier to apply (Westgard,2002), such as:

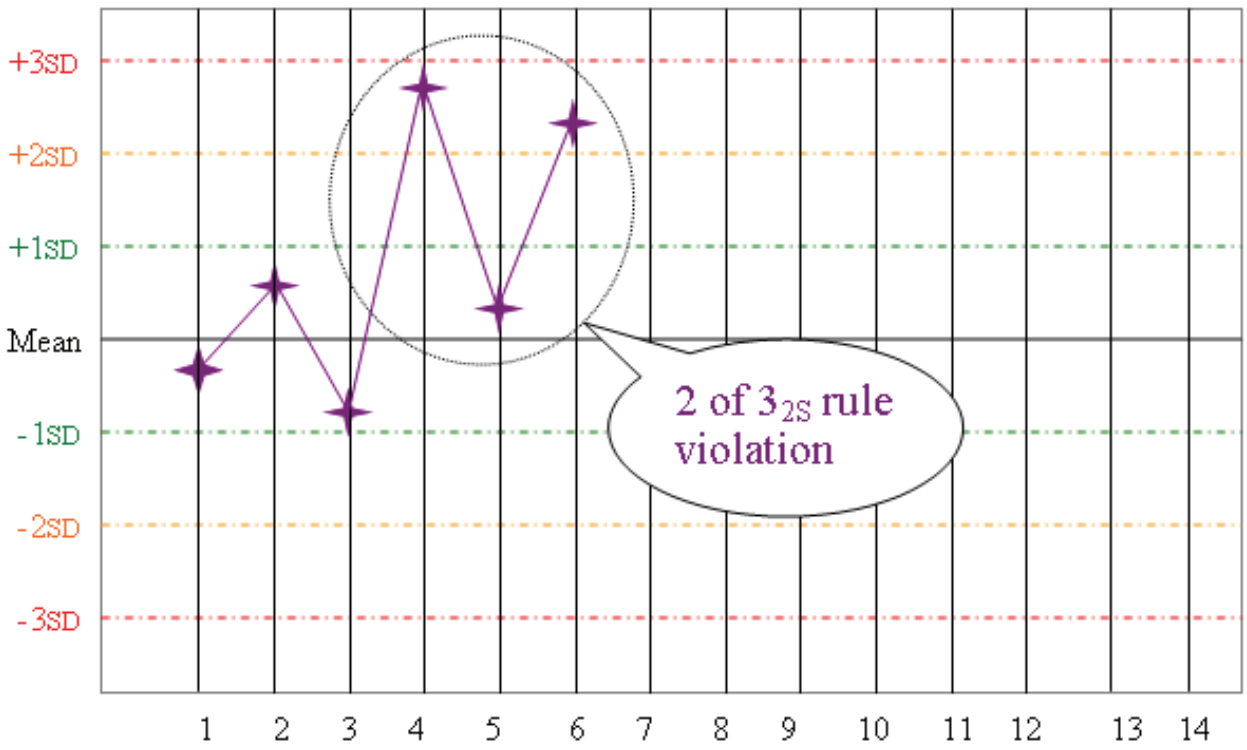

Fig. 9. Rule 2 of $3 z_{2}$ - reject the run when 2 of 3 controls exceed the mean $\pm 2 s$

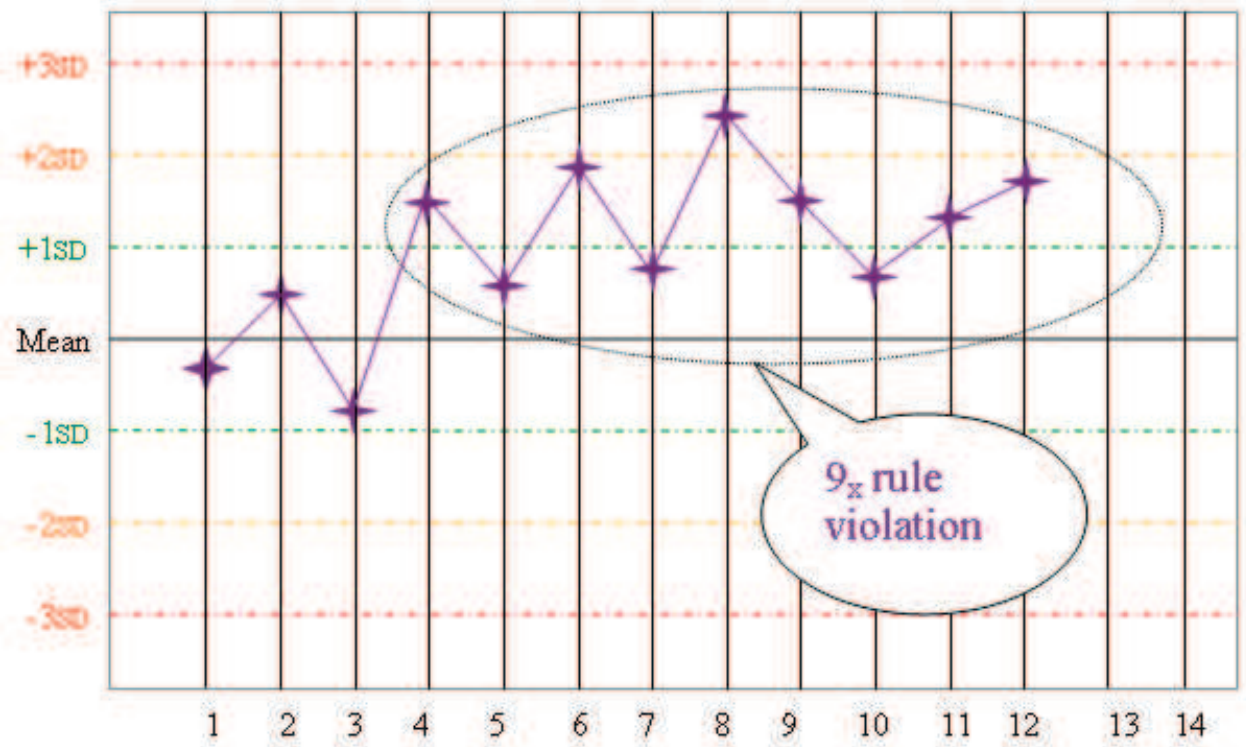

Fig. 10. Rule 9x - reject when 9 consecutive control measurements fall on one side of the mean. 


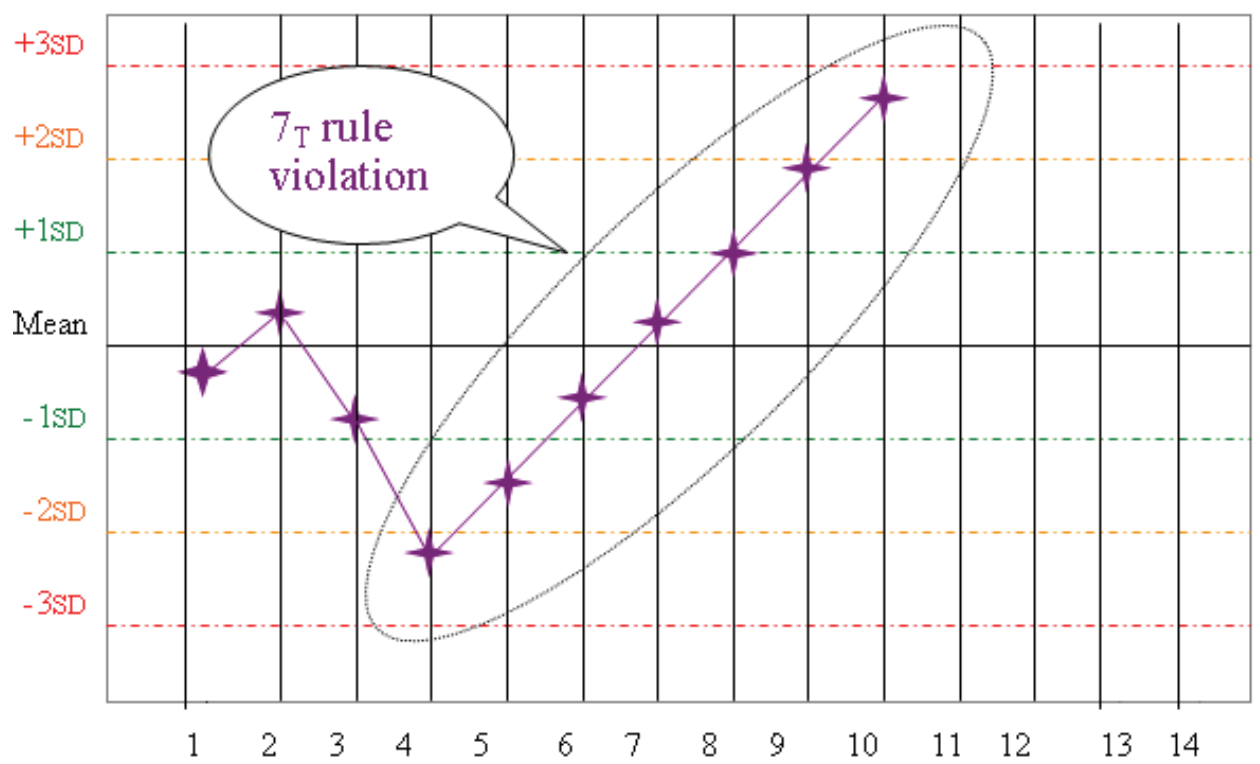

Fig. 11. Rule 7T - reject when seven control measurements trend in the same direction, either higher or lower.

Examples of most commonly used "QC Rule Combinations":

- In Clinical Chemistry tests, the controls are usually run in multiples of 2, hence, the QC combination rule will be: $1_{3 S} / 2_{2 S} / R_{4 S} / 4_{1 S} / 10 x$

- On the other hand in hematology, coagulation, blood gases tests, the controls are usually run in multiples of 3 , hence, the QC combination rule will be: $1_{3 S} / 2$ of $3_{2 S} / R_{4 S}$ $/ 3_{1 s} / 12 x$

\section{Random and systematic errors}

As was mentioned earlier Levy-Jennings charts and Westgard rules help detect random and systematic errors in a run (BioRad,1995).

Random Errors are defined as those errors which affect the reproducibility or precision of a test system. They are detected by the use of $1_{3 \mathrm{~s}}$ or $R_{4 \mathrm{~s}}$ rules and can be due to variations in line voltage, pipettes, dispensers, contamination, volume dispensed, bubbles in lines of reagents, etc.

The other type of errors is the Systematic Errors. Systematic Errors are bias or shifts and Trends in the QC data. These errors affect the accuracy of the test system. They are usually detected by the use of the $22_{2}, 4_{1 s}$, or $10_{x}$ rules and can be due to calibration lot changes, temperature changes in incubator unit, light source deterioration, electronics, reagent lot changes, etc. (BioRad,1995).

\section{Precision}

Precision is the degree of agreement among repeated measurements of the same substance on the same sample, or on separate samples collected as close as possible in time and place. Precision indicates the following:

- How consistent and reproducible the laboratory method is.

- It shows how close the measurements are to each other. 
- It may not mean that the sample results actually reflect the "true" value, but rather it reflects that analysis is giving consistent results under similar conditions.

Precision is verified through the use of duplicate samples or measurements (DerekLaw,2007). Duplicate samples are defined as more than one sample taken from the same source at the same time. Hence, precision is determined by calculating the Standard Deviation (SD) or coefficient of variation of the samples. The smaller the value of the coefficient of variation is (or standard deviation) the more precise the measurements.

\section{Accuracy}

Accuracy is a measure of confidence in a measurement. It indicates how close test results to the true or target value (Derek-Law,2007). The smaller the difference between the test results and its "true" or expected value; the more accurate the measurement will be. The more precise or reproducible the values are, the more reliable or accurate the result. Measurement of accuracy can be determined by comparing a sample that has a known value such as a standard reference material to the technician's measurement of that sample.

Example:

If the $\mathrm{pH}$ readings of a standard buffer were as follows:

7.5, 7.4, 6.7, 7.2, 6.8, 7.3, 6.5, 7.2, 6.8, 7.0, 7.4, 7.2

The mean of these results will be 7.08

Accuracy $($ bias $)=$ mean value - true value $(7.08-7.0)=0.08$

Therefore, the difference is biased by +0.08 units. The bias reflects the error in comparison to the mean. Figure 12 illustrates the difference between precision and accuracy (DerekLaw,2007).

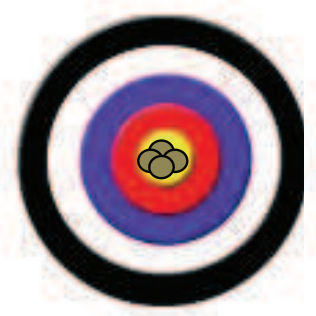

High Accuracy High Precision

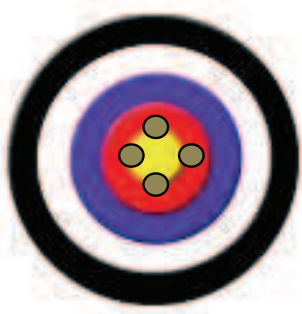

High Accuracy Low Precision

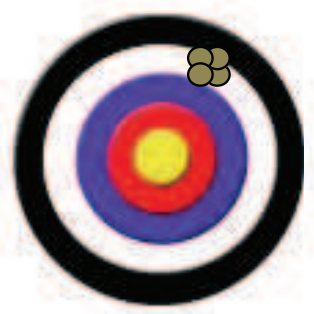

Low Accuracy High Precision

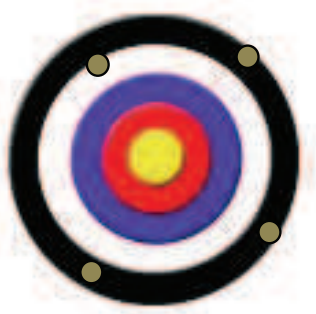

Low Accuracy Low Precision

Fig. 12. A diagrammatic representation of the difference between accuracy and precision.

\subsubsection{Computer Software for Assessing QC validation}

QC programs were developed to facilitate and accelerate the implementation of QC rules before reporting patient's results. There are three commercially available programs that help in the selection of QC procedures and they are listed as follows (Westgard,2002):

- $\quad$ Validator ${ }^{\circledR} 2.0$ by Westgard QC, Inc., http://www.westgard.com/lesson2.htm

- Quality Advisor by Quality Is Key, Inc., http://www.qualityiskey.com

- $\quad$ EZ Rules® by Westgard QC, Inc. http://www.westgard.com/ezrules.htm

Some of the currently available QC software that could be used for industrial applications and that software may be adapted for laboratory applications are summarized below (Westgard,2002).

- $\quad$ Bio-Rad Unity (http://www.bio-rad.com). 
- MAS/Dade LabLink (http://www.mas-inc.com).

- Hematronix Real-Time QC (http://www.hematronix.com) is primarily a "real-time" peer-comparison service.

- Sigma Diagnostics Computrol on Line (http://www.sigma-aldrich.com)

- Fisher Scientific ConCurTRAK (http://www.fishersci.com

- Boston Biomedica AccuChart (http://www.bbii.com) is an internal QC program for specialized tests in infectious disease.

- Blackhawk Biosystems Virotrol QA (http://www.blackhawkbiosystems.com)

- Westgard EZ Runs TM (http://www.westgard.com)

However, most of these programs are complex, expensive, or designed for specialized tests, or available only upon purchasing the company's control materials. Therefore, we decided to design our own QC software keeping in mind the most important features required in a QC program such as it should be straightforward and user-friendly, consists of a rapid setup for new applications, capable of easily adjusting the mean and SD according to the inhouse lab values, the program should be capable of plotting the manufacturer QC mean and $\mathrm{SD}$ at the beginning of a new lot. Thereafter, the program must be capable of automatically updating the mean and SD values after accumulating minimum 20 points. In addition, the program must be capable of representing the QC data in charts such as the Levy-Jennings chart with the option of displaying multilevel, multistage, single chart display. Finally, the program should be equipped with an automatic flagging when a $\mathrm{QC}$ rule is violated and capable of generating exception or exclusion logs or reports. The best QC program will be capable of exporting the QC data and comparing it to peer-review data (Westgard,2002). The unique feature in our QC software is that in addition to the features mentioned above it is capable of monitoring work progress, report generation, and classification of system end users corresponding to access privileges. Figure 13 shows a physical layout for the clientserver architecture of the proposed QC system. The main advantages of this architecture are its simplicity, low-cost and seamless compatibility with existing systems (Elmasri,2003). However, when the work environment is distributed over a wide geographical area, this architecture can be upgraded to be completely web-based application (Al-Taee,2009).

The software architecture that we developed is explained in details in our paper (AlTaee,2009). As for the QC rules used in our newly designed program we implemented the following rules only as the program is used mainly for the serology laboratories. $\mathbf{1}_{3 \mathrm{~s}} / \mathbf{R}_{\mathbf{4 s}} / \mathbf{2}_{\mathbf{2 s}}$ $/ 4_{1 s} / 1_{2 s}$.

The reporting process in our QC program aimed at preparing and printing various types of reports. The contents of these reports vary depending on the user type. For example, the technicians are allowed to prepare and print reports related to the tests they perform while the director can prepare reports on all tests and users existing in the system database (AlTaee,2009).

Several immediate improvements were evident through using our developed QC system. Although detailed data is not yet available; table 3 shows initial extrapolated estimates for performance compared with the original paper-based system. Furthermore, the developed QC system is capable of generating reports summarizing personnel and instrument performance by reporting the number of times the QC was repeated, the reasons for this repetition as well as how frequent the technicians was running the controls as a measure of competency and evaluation (Al-Taee,2009). 


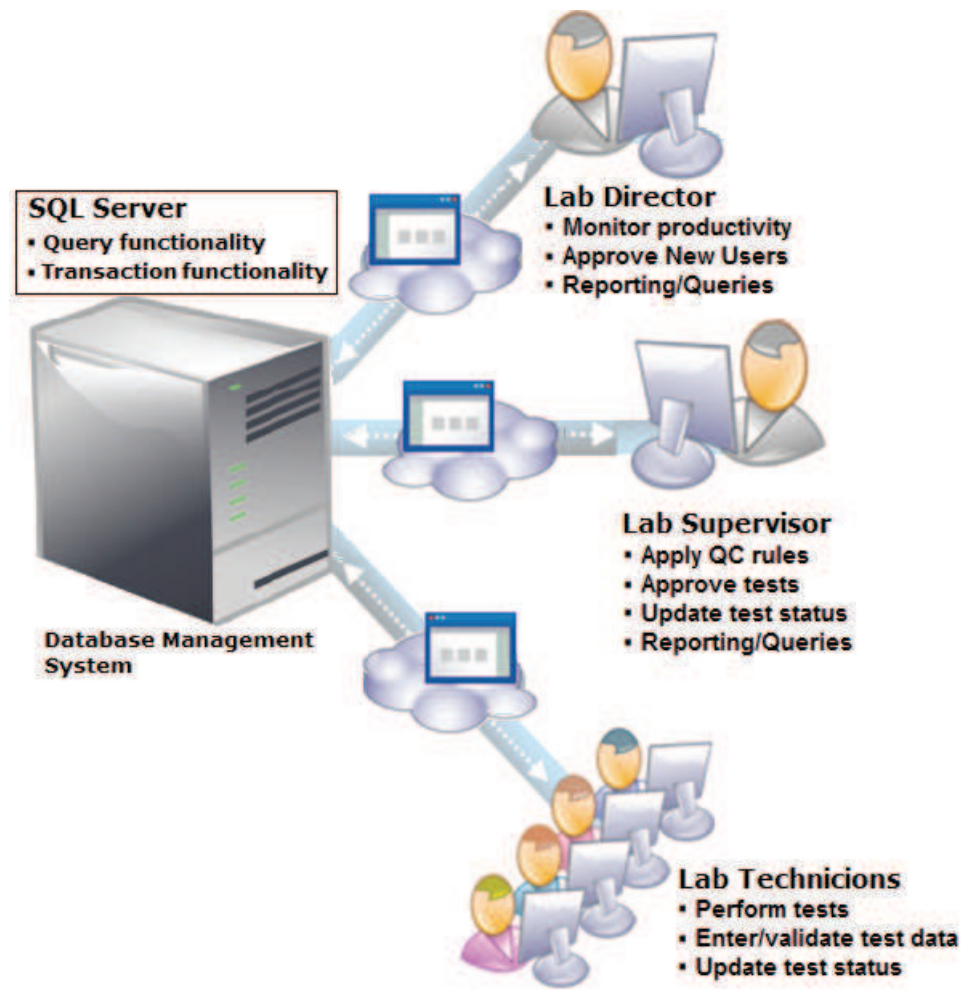

Fig. 13. The client-server architecture of the QC system.

\begin{tabular}{|l|l|l|}
\cline { 2 - 3 } \multicolumn{1}{c|}{} & \multicolumn{2}{c|}{ Estimated Processing Time } \\
\cline { 2 - 3 } \multicolumn{1}{c|}{} & $\begin{array}{l}\text { Web-based } \\
\text { System }\end{array}$ & $\begin{array}{l}\text { Paper-based } \\
\text { System }\end{array}$ \\
\hline Place test & $<2 \mathrm{~min}$ & $5 \mathrm{~min}$ \\
\hline Approve test & $<1 \mathrm{~min}$ & $15 \mathrm{~min}-1$ hour \\
\hline Apply Westgard rules & $<1 \mathrm{~min}$ & $15 \mathrm{~min}-1$ hour \\
\hline View work productivity & $<2 \mathrm{~min}$ & $14 \mathrm{~min}-1$ hour \\
\hline Prepare annual test report & $<5 \mathrm{~min}$ & $1-2$ days \\
\hline
\end{tabular}

Table 3. Comparison between the developed and paper-based QC systems.

In conclusion, the QC prototype system which we developed was tailored for the specific quality control plan of the serology tests at medical laboratories; however, it can be extended to other sections of clinical laboratories. Standardizing the QC processes on one system ensured that all clinical tests are evaluated using common quality requirements and appropriate QC rules. Furthermore, it provided better processing for the documentation of new reagent/calibrator and QC lot numbers as well as faster, enhanced and more accurate information provided to management to respond to and document $\mathrm{QC}$ issues. The control 
material for each test is processed in the lab and the results are used for monitoring the accuracy of analytical methods. Such information provides analysts with confidence that results acquired from actual samples are correct. Moreover, the developed system provides daily, weekly and monthly reporting on-line for management as well as easy tracking of proficiency testing performance and problem resolution. Finally, the adopted objectoriented development approach allows for easy maintenance and expansion and therefore more QC options can be added in the future. The innovative approach in our QC software was that it allows the medical director to monitor QC performance as well as instrument performance and technician performance due to its ability to generate reports summarizing the failures in QC classified by its origin whether it was due to instrument malfunction or technician negligence. Reviewing and carefully analyzing these reports helps keep close monitoring of clinical tests performed in the laboratory and aids in initializing quality improvement projects for failed QC. However, further work is still needed to refine this project to display the proposed corrective action(s) taken when QC values are out of control (Al-Taee,2009).

\subsubsection{Proficiency testing}

In addition to performing internal QC, it is important to participate in External Proficiency testing (Tholen,2008). Some of the advantages of participating in external quality assessment are the following:

- These programs enable laboratories to compare their performance in terms of accuracy and precision with those of their peers by analysing the same samples using their routine analytical methods, which are prepared and circulated by the scheme organisers. The results obtained by all the laboratories are analysed and compared with a reference value for the determinants by the scheme organisers and the laboratory performance is reported back to the participants.

- External Proficiency testing is a key requirement of all accreditation bodies.

- Proficiency testing helps to highlight reproducibility performance between laboratories and helps is detecting systematic errors such as bias in data reported.

\subsubsection{Quality Assurance in the post-analytical stage}

Quality Assurance does not end by getting the results from the instrument. The post analytical stage is very critical in assuring accurate results. This section demonstrates two types of the post-analytical checks, Technical and Administrative checks.

\subsubsection{Technical Checks}

Technical checks involve the following.

- Clinical Correlation: This is the correlation of patient's test result with the clinical diagnosis.

- Correlation with other laboratory tests: Under certain circumstances the combinations of the result of one test with other results become impossible due to discrepancy between the results. Hence, if checks for correlation between the results are made before reporting the results are made, errors can be identified and corrected before results are released to patient's file. For example, it is unusual to see $4+$ bacteria by urinalysis microscopy and no growth in culture.

- Delta Checks: Delta checks refer to test results that fall outside certain clinically significant limits or critical values. Hence, the result will fail or will be flagged to alert 
the technician to review the result before releasing it. Another purpose of the delta check is to compare the results of the patient with the previous result of the same test done on more than one occasion. In this case delta check or variation between the previous test results can be compared to see if they fall within the limit allowed for normal changes.

- Normal range check or limit Check: In this check the patient's results are checked if they are impossible or incompatible with the normal range of the test. Impossible results may be due to clerical errors, short sampling or clot formation, improper identification of fluid being analyzed, improper specimen identification or improper sample collection.

Technical checks usually depend on education, training, experience and technical skill of individuals performing the tests. The technologist should be qualified enough to handle and interpret test results.

\subsubsection{Administrative Checks}

Administrative Checks involve reporting results to physicians. There are several areas that should be closely monitored to ensure accurate reporting of patient's results. Some of the areas are as follows:

- Reporting Mechanism: Some problems may arise in reporting results due to: Organization and format of report, flagging of abnormal results, reporting of critical results by telephone, delay in posting results in medical records, reporting on or after the discharge of patient.

- $\quad$ Record Keeping: Refers to the mechanism of maintenance of records related to patient results. The importance of record keeping is to be able to refer back to previous results to check patient history. Record keeping can be accomplished by the following: Paper records, computer technology, microscopic slide from patient specimens, and tissue blocks from pathology specimens.

- Test Completion Check: This check is to verify that all tests ordered are completed before releasing the results. This step is critical in order to provide a complete picture for the diagnosis. However, some results may be delayed because the test was sent to a referral laboratory, therefore, the results must be filed in an organised secure folder until all test results are ready to be released. Another scenario if the physician desires to add another test at a later stage to verify a certain diagnosis, therefore, proper storage of the original patient-sample must be done in order to assure fast retrieval of the sample to perform the test especially if it was a precious sample such as CSF (cerebrospinal fluid) or bone marrow sample.

- $\quad$ Reflex Testing Protocol: This refers to the situation when a single test result may not provide the physician with enough information to determine diagnosis; hence, another test is automatically performed by the lab without the treating physician's request. For example, serum protein electrophoresis is usually accompanied by immunofixation in order to accurately diagnose the type of the multiple myeloma bands or clone.

\section{Performance improvement}

Performance Improvement is a process which uses information from multiple sources to increase the probability of accomplishing desired outcomes to better serve the needs and expectations of patients. The most frequently used Performance Improvement tools are: 1. 
FOCUS PDCA - framework for improvement, 2. Failure Modes and Effects Analysis (FMEA), 3. Root Cause Analysis (RCA) and 4. Control Charts which we discussed earlier in this chapter. In this section of the chapter we will focus on one model of quality improvement approaches which is "FOCUS PDCA " and give examples on its implementation in a clinical laboratory setting.

Quality assurance (QA) and its component, Quality Improvement (QI), began in the engineering and manufacturing fields where statistical process control and continuous quality improvement were combined with general management methods. Since then QA and QI were combined and implemented in healthcare systems.

Quality Assurance activities are often represented as three points on a QA triangle (Figure 14). The points are: defining quality (QD), measuring quality (QM), and improving quality (QI). QD refers to developing outcome standards that the healthcare delivery system must meet in order for its patients to achieve the best health gains. QM consists of quantifying the current level of compliance with standards and finally, QI represents the use of appropriate methodologies to close the gap between the current and expected levels of quality. Quality Improvement often uses quality management tools and principles to recognize and deal with system inefficiencies (Bornstein,2001).

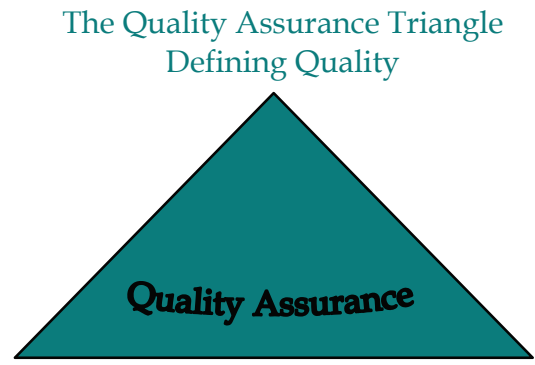

Improving Quality

Measuring Quality

Fig. 14. The Quality Assurance Triangle

Approaches to conducting QI activities are numerous; one of these activities is the Performance Improvement Model FOCUS-PDCA.

The FOCUS phase helps to narrow the team's attention to a distinct opportunity for improvement (Bader,2003).

The letter " $F$ " stands for FIND. Find a process that needs improvement and describe the process of the improvement plan.

The letter "O" stands for ORGANIZE. Choose a team who is knowledgeable in the process. Determine the team size and members and try to choose a representative from each level in the organization, select members, and be ready to document their progress.

The letter "C" stands for CLARIFY. Highlight and clarify the current knowledge of the ongoing process.

The letter "U" stands for UNDERSTAND. Understand the causes of variation. The team will be responsible for measuring the process and learning the causes of variation which will help them in preparing a plan for collecting data as well as establishing specific, measurable, and controllable variations. 
The letter "S" stands for SELECT. Select the possible process for improvement and determine the action(s) that need to be taken to improve the current process.

The P-D-C-A phase allows the team to follow that opportunity and review its outcome.

The letter " $\mathrm{P}$ " stands for P PLAN. Plan the improvement by investigating the process, choosing possible steps for improvement, and identify which data would be helpful to collect that might aid in the improvement process.

The letter " $\mathrm{D}$ " stands for DO. Do the improvement/data collection/data analysis and carry out a simulation of the correction plan on a small-scale.

The letter "C" stands for CHECK. Check the data for process improvement and document the results of the change. Modify the change, if necessary and possible.

The letter "A" stands for ACT. Act to hold the gain or continue the improvement process and carry out the change if it is working. However, if the performance improvement plan fails, abandon the plan and repeat the cycle (Bader,2003).

\section{Conclusion}

In this chapter, we have demonstrated the latest approaches in quality control and quality management in clinical laboratories. Furthermore, we highlighted our recent work in which we constructed new quality control software which is tailored for the specific quality control plan for the immunology/ serology tests at medical laboratories. This program is capable of standardizing the QC processes in one system which ensures that all clinical tests are evaluated using common quality requirements and appropriate $\mathrm{QC}$ rules. Furthermore, it provides better processing for the documentation of new reagent/calibrator and QC lot numbers.

In addition, this program grants faster, enhanced and more accurate information to the management in order to respond to and document QC issues. Control material for each test is processed in the lab and the results are used for monitoring the accuracy of analytical methods. Such information provides technologists with confidence that results acquired from actual samples, are correct. Moreover, the developed system provides daily, weekly and monthly reporting on-line for management as well as easy tracking of proficiency testing performance and problem resolution. The uniqueness of our software resides in the reports generated by the program. For example, the QC failure reports are linked to the performer which gives the director a chance to assess the competency of the employees performing the tests as well as the efficiency of the analytical instruments. Furthermore, we have demonstrated how the benefits provided by the improved QC program accelerated the quality assurance process of the tests performed. Finally, the adopted object-oriented development approach that we used allows for easy maintenance and expansion and therefore more QC options can be added in the future. Further work is still needed to refine this QC software to display the proposed corrective action(s) taken when QC values are out of control.

\section{References}

Al-Taee, M., Souan, L., Al-Haj, A., Mohsen, A. and Muhsin, Z. (2009 of Conference). Quality Control Information System for Immunology/ Serology Tests in Medical Laboratories. 6th International Multi-Conference on Systems, Signals and Devices. ISBN, Djerba - Tunisia, 23-26 March 2009 Published, 
Bader, M. K., Palmer, S., Stalcup, C. and Shaver, T. (2003). Using a FOCUS-PDCA quality improvement model for applying the severe traumatic brain injury guidelines to practice: process and outcomes. . Evid. Based Nurs. 6, (6-8),

BioRad. (1995). Basic E Intermediate Systems of Quality Control for the Clinical Laboratory.BioRad Laboratories, Lit. No. Q-1102 4/05,

Bornstein, T. (2001). Quality Improvement and Performance Improvement: Different Means to the Same End? QA Brief. Volume 9, (6-12),

Derek-Law, E. (2007). Accuracy Versus Precision.Geodatabases That Are Easier to Set Up and Manage. http://www.esri.com/news/arcuser/0807/gdb_precision.html,

Elmasri, R. a. N., S. B. . (2003). Fundamentals of database systems.Addison Wesley, USA,

Lumsden, J. H. (2000). Laboratory test method validation. Revue Med. Vet. 151, (623-630),

Plebani, M. (2006). Errors in clinical laboratories or errors in laboratory medicine? Clinical Chemistry and Laboratory Medicine. 44, (750-759), 1434-6621

A. J. Reynolds. (2003). Unit 5 Section 2 : Mean, Median, Mode and Range.Mathematics Enhancement Program,

Schieltz, B., and Westover, B.; 2010). Descriptive Statistics Medialab,

Sharma, P. (2009). Preanalytical variables and laboratory performance. Indian Journal of Clinical Biochemistry. 24, (109-110), 0970-1915

Tholen, D. W., Berte, L.M., Boone, D.J., Cooper, W.G., Gun-Munro, J., Nobel, M.A., Sarewitz, S.J., Williams, M.L. (2008). Using Proficiency Testing to Improve teh Clinical Laboratory; Approved Guideline. Clinical and Laboratory Standards Institute (NCCLS). 27, (GP27-A22),

M. Thompson. (2010). Internal quality control in routine analysis.1757- 5958,

Westgard, J. (2009). "Westgard Rules" and Multirules.

Westgard, J. O. (2002). Basic QC Practices.Westgard QC, Inc., 1-886958-17-3, Madison

Westgard, J. O., Miller, W. G., Allen, K., Boone, D. J., Caines, P., Cooper, G., Garber, C., Greenberg, N., Jain, C. P., Linnet, K., Russek-Cohen, E. and Vaks, J. E. (2006). Statistical Quality Control for Quantitative Measurement Procedures: Principles and definitions; Approved Guideline-Third Edition. Clinical and Laboratory Standards Institute. 26, No.25, (1-33), 1-56238-613-1

Westgard, J. O., Miller, W.G., Allen, K., Boone, D.J., Caine, P., Cooper, G., Garber, C., Greenberg, N., Jain, C.P., Linnet, K., Russek-Cohen, E., Vaks, J.E. (2008). Statistical Quality Control for Quantitative Measurement Procedures: Principles and Definitions; Approved Guideline. Clinical and Laboratory Standards Institute (NCCLS). 26, (C24-A23),

Wikipedia; 2010). Good Laboratory Practice, 


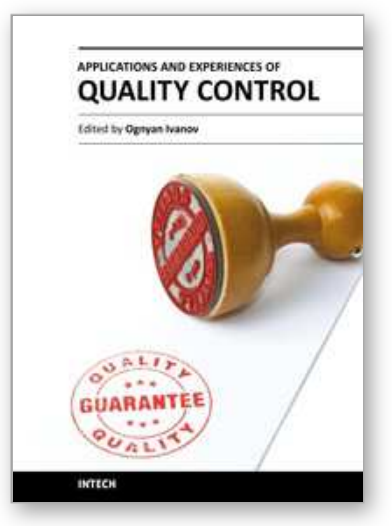

\section{Applications and Experiences of Quality Control \\ Edited by Prof. Ognyan Ivanov}

ISBN 978-953-307-236-4

Hard cover, 704 pages

Publisher InTech

Published online 26, April, 2011

Published in print edition April, 2011

The rich palette of topics set out in this book provides a sufficiently broad overview of the developments in the field of quality control. By providing detailed information on various aspects of quality control, this book can serve as a basis for starting interdisciplinary cooperation, which has increasingly become an integral part of scientific and applied research.

\section{How to reference}

In order to correctly reference this scholarly work, feel free to copy and paste the following:

Lina Souan, Maher A. Sughayer (2011). Innovative Approaches in Quality Management in Clinical Laboratories, Applications and Experiences of Quality Control, Prof. Ognyan Ivanov (Ed.), ISBN: 978-953-307236-4, InTech, Available from: http://www.intechopen.com/books/applications-and-experiences-of-qualitycontrol/innovative-approaches-in-quality-management-in-clinical-laboratories

\section{INTECH}

open science | open minds

\author{
InTech Europe \\ University Campus STeP Ri \\ Slavka Krautzeka 83/A \\ 51000 Rijeka, Croatia \\ Phone: +385 (51) 770447 \\ Fax: +385 (51) 686166 \\ www.intechopen.com
}

\author{
InTech China \\ Unit 405, Office Block, Hotel Equatorial Shanghai \\ No.65, Yan An Road (West), Shanghai, 200040, China \\ 中国上海市延安西路65号上海国际贵都大饭店办公楼405单元 \\ Phone: +86-21-62489820 \\ Fax: +86-21-62489821
}


(C) 2011 The Author(s). Licensee IntechOpen. This chapter is distributed under the terms of the Creative Commons Attribution-NonCommercialShareAlike-3.0 License, which permits use, distribution and reproduction for non-commercial purposes, provided the original is properly cited and derivative works building on this content are distributed under the same license. 